\title{
Caracterização de um Pré-Impregnado Aeronáutico por FT-IR e Análise Térmica
}

\author{
Vanesa C. G. M. Ferrari \\ Departamento de Engenharia Aeronáutica e Mecânica, ITA \\ Faculdade de Engenharias, Arquitetura e Urbanismo, UNIVAP \\ Vera L. Lourenço, Rita de C. L. Dutra, Milton F. Diniz, Margarete F. P. Azevedo, Luis H. David \\ Divisão de Química, ITA
}

\begin{abstract}
Resumo: Este trabalho consiste na caracterização de um pré-impregnado ("prepreg”) de resina epoxídica/fibra de carbono, usando-se espectroscopia no infravermelho com transformada de Fourier (FT-IR), análise termogravimétrica (TG), calorimetria exploratória diferencial (DSC) e análise térmica dinâmico-mecânica (DMTA). A análise por FT-IR foi realizada nos modos de transmissão (pastilha de $\mathrm{KBr}$, pirólise em bico de Bunsen e controlada) e detecção fotoacústica (PAS). Os espectros de FT-IR de transmissão revelaram a presença de resina epoxídica, grupos ciano, amínicos e bisfenol A, que possibilitaram identificar o provável agente de cura:a cianoguanidina (ou dicianodiamida) do sistema epoxídico. Os espectros de FT-IR/PAS permitiram acompanhar as alterações espectrométricas causadas pela cura. A análise térmica auxiliou na observação e compreensão dos eventos durante o processo de cura, etapas de gelificação e vitrificação, e da influência destas nas temperaturas de transição vítrea ( $\mathrm{Tg}$ ) do material curado e na escolha do intervalo de temperatura de cura, que é um dos parâmetros mais importantes do processo produtivo.
\end{abstract}

Palavras-chave: Caracterização, compósito, resinas epoxídicas, ciclo de cura, FT-IR, análise térmica.

\section{Characterization of Pre-Impregnated of Epoxy Resin/Carbon Fiber}

Abstract: This work explores the characterization of pre-impregnated ("prepreg") materials made with an epoxy resin/carbon fiber, using FT-IR spectroscopy, thermogravimetry (TG), differential scanning calorimetry (DSC) and dynamic mechanical thermal analysis (DMTA). FT-IR spectroscopy was used in the transmission mode ( $\mathrm{KBr}$ pellets, pyrolysis without control and controlled pyrolysis) and photoacoustic detection (FT-IR/PAS). The transmission FT-IR spectra revealed the presence of epoxy resin, cyano groups, amine and bisphenol A, which allowed us to identify the probable agent of cure: cyanoguanidine (or DCD). With FT-IR/PAS it was possible to monitor spectrometric changes caused by curing. The thermal analysis assisted in observing and understanding events during the curing process, including the gelation and vitrification steps. It was also useful to study the influence in the glass transition temperature (Tg) of the cured material and for choosing the curing temperature range, which is one of the main parameters in the manufacturing process.

Keywords: Characterization, composite, epoxy resin, cure, FT-IR, thermal analysis.

\section{Introdução}

Os compósitos poliméricos à base de resina epoxídica são escolhidos preferencialmente, para aplicações aeronáuticas e espaciais devido à relação entre custo, peso, propriedades térmicas e mecânicas. Assim, é de vital importância conhecer e monitorar o ciclo de cura e correlacionar estas informações com as propriedades mecânicas e microestruturais finais para a otimização do processamento.

Muitas das tecnologias modernas requerem materiais que apresentem combinação de propriedades não convencionais, as quais não podem ser encontradas em ligas metálicas, materiais cerâmicos ou poliméricos. A indústria aeronáutica, em particular, está em permanente busca de materiais estruturais que apresentem baixa densidade, melhor relação de propriedades mecânicas/peso e resistência à abrasão ao impacto ${ }^{[1]}$. Este desenvolvimento está relacionado com a formulação das resinas presentes nos compósitos, o tipo de polímero, o agente de cura e, até mesmo, a razão estequiométrica resina/agente de cura, que influenciam diretamente as propriedades mecânicas do compósito.

As resinas termorrígidas, embora sejam frágeis à temperatura ambiente e não possam ser moldadas novamente sob aquecimento, devido às fortes ligações químicas, diferentemente das resinas termoplásticas, possuem maior valor de módulo e resistência à tração, excelente resistência química, estabilidade dimensional e térmica, boa resistência à fluência e melhores propriedades em fadiga. Também são facilmente processadas devido à baixa viscosidade. Logo, são usadas preferencialmente no processamento de compósitos estruturais.

As resinas termorrígidas mais comuns para esse fim são as epoxídicas, as poliésteres insaturadas e as fenólicas ${ }^{[2]}$.

O termo "prepreg" é utilizado pela indústria de material compósito para identificar reforços de fibras pré-impregnadas com uma resina polimérica parcialmente curada. Este material é enviado ao cliente na forma de uma fita ou tecido, o qual é moldado e curado sem adicionar nenhum outro produto ou resina. Esta provavelmente, é a forma que o material compósito é amplamente utilizado para a fabricação de peças com aplicações estruturais, especialmente na indústria de produtos para o mercado aeroespacial.

É bem conhecido que as propriedades da formulação de um sistema epoxídico, isto é, o tipo de agente de cura (endurecedor) e a razão estequiométrica resina/endurecedor, influenciam as propriedades mecânicas do compósito ${ }^{[3]}$. Portanto, o conhecimento de agentes de cura é importante para alcançar as propriedades desejadas. Pesquisas envolvendo a caracterização do comportamento

Autor para correspondência: Vanesa C. G. M. Ferrari, Faculdade de Engenharias, Arquitetura e Urbanismo, Universidade do Vale do Paraíba - UNIVAP, Av. Shishima Hifumi, 2911, Urbanova, CEP 12244-000, São José dos Campos, SP, Brasil, e-mail: vanesa_mitchell@yahoo.com.br 
de cura de resinas epoxídicas, por meio de dados térmicos ou de caracterização por espectroscopia com transformada de Fourier (FT-IR) de agentes de cura utilizados em resinas epoxídicas, entre outros, vêm sendo desenvolvidas por diferentes pesquisadores da comunidade científica ${ }^{[3-6]}$.

Sugita e Ito $(1965)^{[5]}$ demonstraram que agentes de cura carbonilados são mais facilmente identificáveis, desde que na análise da amostra na forma de pastilha de $\mathrm{KBr}$, a presença de grupo $\mathrm{C}=\mathrm{O}$ de éster, resultante da reação de cura do anel epóxido/anidrido, seja visualizada. Entretanto, para a separação do agente de cura, foram utilizadas metodologias complexas, com várias etapas.

Romão et al. $(2001)^{[7]}$ e Dutra (1997) ${ }^{[8]}$ demonstraram a separação e caracterização por FT-IR de agentes de cura do tipo anidrido em resina epoxídica e do tipo amido-amina em compósitos, com metodologia simples e rápida, envolvendo técnicas de pirólise controlada FT-IR (CONTROLPIR/FT-IR), mesmo neste último sistema, onde era esperado um grau de dificuldade maior por tratar-se de compósito.

Concluiu-se ${ }^{[7,8]}$ que a técnica CONTROLPIR/FT-IR, que analisa o produto líquido resultante da degradação da amostra aplicada a estes sistemas, em condições adequadas, apresenta em relação às técnicas FT-IR convencionais, a vantagem de separar o agente de cura. Isto permite a sua caracterização, independentemente de sua função orgânica básica ser indicada ou não na análise da amostra curada.

Em trabalho recente ${ }^{[4]}$ amostras de resina epoxídica (EP) curadas com compostos à base de polimercaptana $(\mathrm{SH}), \mathrm{SH}$ na presença de poliamino amida e amina modificada constituindo, respectivamente, os sistemas epoxídicos (SE) 1, 2 e 3, foram preparados segundo condições estabelecidas pelas curvas de calorimetria exploratória diferencial (DSC) de EP e agentes de cura ou endurecedores (AC) e analisados, antes e após preparação do SE, por FT-IR por meio de técnicas de transmissão (pastilha de $\mathrm{KBr}$, pirólise por bico de Bunsen, e pirólise controlada (CONTROLPIR/FT-IR) dentro da faixa de temperatura fornecida pela análise termogravimétrica (TG) dos SE 1, 2 e 3, para a caracterização de cada AC. Para tal fim, as absorções FT-IR do pirolisado líquido obtido pela metodologia CONTROLPIR/FT-IR foram avaliadas, em comparação ao espectro do AC de referência. A caracterização dos agentes de cura foi feita com a técnica TG/FT-IR do gás liberado de cada SE, em comparação ao espectro de referência do produto de degradação do respectivo AC.

Sales et al. (2010) ${ }^{[6]}$ estudaram a cura de "prepregs" por espectroscopia de luminescência, comparando dados obtidos com os relativos à análise por FT-IR, na região do infravermelho próximo (NIR). Os resultados obtidos por ambos os métodos indicaram que a reação de cura pode ser monitorada pelas alterações espectrométricas observadas durante o processo.

O mecanismo de reticulação das resinas termorrígidas, principalmente as epoxídicas, é muito complexo devido à interação entre a cinética química e as mudanças das propriedades físicas ${ }^{[2]}$, o que levou ao desenvolvimento de inúmeros e, por vezes, contraditórios modelos para prever os ciclos de cura ${ }^{[9]}$.

A utilização de técnicas de Análise Térmica para avaliar o comportamento de cura de resina epoxídica tem sido ampla e, em especial DSC e DMTA têm fornecido muita informação sobre o mecanismo isoladamente ${ }^{[9-14]}$ ou em compósitos ${ }^{[10,15-25]}$.

A técnica DSC mede diretamente a extensão e razão de conversão durante a cura, porém, quando a velocidade de cura diminui e afeta a sensibilidade de medição, os eventos relacionados às alterações reológicas durante a cura, como a gelificação e a vitrificação, não são observados por esta técnica ${ }^{[10,13]}$.

A análise DMTA apresenta baixo nível de detecção na região de temperatura localizada abaixo do ponto de gelificação, onde o módulo elástico é muito baixo, porém, é sensível às grandes mudanças que ali ocorrem a partir do ponto de gelificação até o final da reação, incluindo a vitrificação. Assim, as características de cura de resinas termorrígidas, tais como a velocidade de cura, o ponto de gel ou de vitrificação, o tempo de cura e os efeitos das condições de processamento, podem ser obtidos pela medição dos módulos elástico e de perda em função da temperatura, do tempo, ou ambos ${ }^{[10,13,20,26]}$. Além disso, a análise DMTA apresenta melhor precisão e sensibilidade em comparação com os resultados obtidos por DSC, por exemplo, na determinação da Tg de materiais com alto grau de cura ou materiais reforçados ${ }^{[24,26]}$.

Portanto, as análises DMTA e DSC são complementares no estudo de cura dos materiais termorrígidos.

O presente trabalho apresenta a caracterização do "prepreg" de resina epoxidica/fibra de carbono por meio das análises FT-IR e pelas técnicas, análise termogravimétrica (TG), calorimetria exploratória diferencial (DSC) e análise térmica dinâmico-mecânica (DMTA), visando contribuir para o melhor entendimento do processo de cura de sistemas epoxídicos usados em materiais compósitos.

\section{Experimental}

Foram utilizados dois tipos de "prepregs" de uso aeronáutico com a mesma matriz de resina epoxídica, porém, com diferentes tipos de reforços, fibra de carbono (V01X) e fibra de vidro (V02X), este apenas como referência para quantificar o teor de resina no V01X.

Para as análises, foram utilizados os seguintes equipamentos:

- Espectrofotômetro FT-IR Spectrum 2000, Perkin Elmer. Para algumas análises FT-IR foi, também, usado o detector fotoacústico (PAS); e

- Calorímetro Exploratório Diferencial, DSC-910, Analisador Termogravimétrico TGA-951, Analisador Térmico DinâmicoMecânico DMA-983 (todos do fabricante "Du Pont" com o Analisador Térmico TA2100 TA Instruments).

\section{Caracterização do Material}

Para a análise por FT-IR, as amostras foram analisadas segundo a técnica de transmissão (pastilhas de $\mathrm{KBr}$, preparadas na seguinte proporção: $1 \mathrm{mg}$ de amostra para $400 \mathrm{mg}$ de $\mathrm{KBr}$ ). As condições de uso foram: região de 4000 a $400 \mathrm{~cm}^{-1}$, resolução $4 \mathrm{~cm}^{-1}$, ganho 1 e 40 varreduras. A calibração foi feita por meio da análise direta do filme padrão de poliestireno. Também foi utilizado o detector fotoacústico (PAS) FT-IR para a obtenção de alguns espectros, nas seguintes condições de uso: região de 4000 a $400 \mathrm{~cm}^{-1}$, resolução $4 \mathrm{~cm}^{-1}$, ganho 1 e 40 varreduras, velocidades de $0,05 \mathrm{~cm} / \mathrm{s}$ e $0,1 \mathrm{~cm} / \mathrm{s}$. O acessório PAS é calibrado com negro de fumo (MTEC). O procedimento para a investigação do tipo de agente de cura aplicado neste caso, foi a análise sob a forma de pastilha de $\mathrm{KBr}(0,8 \mathrm{mg}$ de $\mathrm{KBr})$, pirólise com auxílio de bico de Bunsen ou pirolisador em diferentes condições, de acordo com o sistema. Os pirolisados líquidos foram analisados como filmes líquidos, também tratados com metanol e/ou água, a quente, e analisados como filmes líquidos ou pastilhas de $\mathrm{KBr}$.

As análises DSC foram realizadas em porta amostra de alumínio sob nitrogênio, nas razões de aquecimento $5,10,15$ e $20^{\circ} \mathrm{C} / \mathrm{min}$ e, em isotermas, nas temperaturas de $100,110,120,130$ e $140{ }^{\circ} \mathrm{C}$. A $\mathrm{Tg}$ foi obtida no segundo aquecimento, a $20^{\circ} \mathrm{C} / \mathrm{min}$.

As análises DMTA, modo flexão, frequência $1 \mathrm{~Hz}$, amplitude de oscilação $1 \mathrm{~mm}$, foram realizadas a $3{ }^{\circ} \mathrm{C} / \mathrm{min}$ de $25^{\circ} \mathrm{C}$ a $250{ }^{\circ} \mathrm{C}$, ou em isoterma, $100,110,120,130,140$ e $150{ }^{\circ} \mathrm{C}$. 
As análises TG consistiram no aquecimento da amostra a partir de $25{ }^{\circ} \mathrm{C}$ a $20{ }^{\circ} \mathrm{C} / \mathrm{min}$ até $620{ }^{\circ} \mathrm{C}$, isoterma por 40 minutos, sob nitrogênio ${ }^{[27]}$.

\section{Resultados e Discussão}

A análise por FT-IR está baseada na experiência obtida pelo grupo, em um trabalho anterior ${ }^{[4]}$, no qual foi possível separar e caracterizar por FT-IR o tipo de resina epoxídica e o agente de cura presente no compósito.

A Figura 1a e a Tabela 1 indicam absorções FT-IR características do "prepreg" V01X que, associadas ao seu formato, sugerem a presença básica de resina epoxídica, grupos ciano e amínico, o que pode ser confirmado pela comparação com o espectro de resina epoxídica da literatura ${ }^{[28]}$

A Figura 2a apresenta as bandas FT-IR do pirolisado do "prepreg", obtido com o uso do bico de Bunsen e suas principais absorções em regiões características que estão relacionadas na Tabela 2.
A análise por FT-IR sugere a liberação de bisfenol A, matéria-prima da resina epoxídica, e pode ser confirmada pela comparação com o espectro presente na literatura ${ }^{[29]}$.

A Figura $2 \mathrm{~b}$ apresenta as bandas do pirolisado do "prepreg" obtido com o pirolisador a $340{ }^{\circ} \mathrm{C} / 10 \mathrm{~min}$, método que permite evidenciar o agente de cura ${ }^{[4]}$, devido ao controle de temperatura e tempo. Suas principais absorções estão relacionadas na Tabela 3 e sugerem a presença básica de resina epoxídica, grupos ciano, amínicos e/ou amídicos.

A resina do "prepreg" foi extraída com metanol a quente, visando à separação de compostos contendo $\mathrm{C} \equiv \mathrm{N}$. O resíduo obtido foi analisado e apresentado no espectro da Figura 3a; as principais absorções características estão na Tabela 4. Este conjunto de dados sugere a presença de resina epoxídica e evidencia melhor as absorções de cianoguanidina (ou dicianodiamida), na região de 3380 a $2000 \mathrm{~cm}^{-1}$ (grupos amino e ciano), que pode ser comparado ao espectro de cianoguanidina presente na literatura ${ }^{[30]}$.

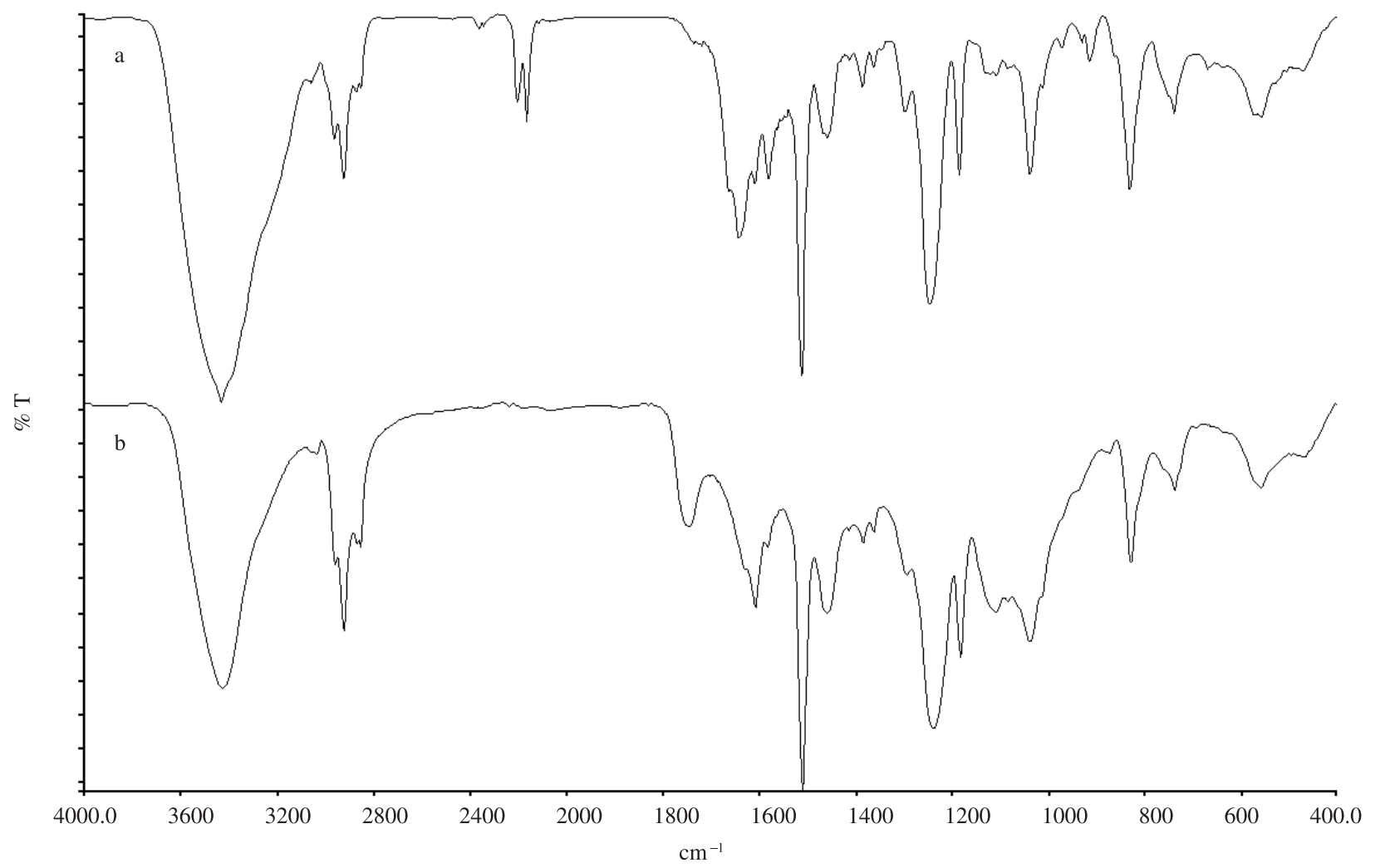

Figura 1. Espectros FT-IR do "prepreg" V01X em pastilha de KBr: a) sem cura e b) após a cura a $120{ }^{\circ} \mathrm{C}$ em DMTA.

Tabela 1. Bandas características do "prepreg" V01X, atribuídas aos modos vibracionais típicos (Figura 1a).

\begin{tabular}{|c|c|c|c|}
\hline $\mathrm{N}^{0}$ de onda $\left(\mathrm{cm}^{-1}\right)$ & Atribuição & $\mathrm{N}^{\circ}$ de onda $\left(\mathrm{cm}^{-1}\right)$ & Atribuição \\
\hline 2964 & $\mathrm{v}_{\mathrm{a}} \mathrm{CH}_{3}$ & 1458 & v C-C aromático \\
\hline 2925 & $v_{\mathrm{s}} \mathrm{CH}_{3}$ & 1385 & $\begin{array}{c}\delta \mathrm{CH}_{2} \\
\delta \mathrm{s} \mathrm{CH}_{3}\end{array}$ \\
\hline 2204 & $\nu \mathrm{C} \equiv \mathrm{N}$ & 1245 & v C-O éter \\
\hline 2165 & $\vee \mathrm{C} \equiv \mathrm{N}$ & 914 & Grupo epoxy terminal \\
\hline 1640 & $\delta \mathrm{NH}_{2}$ & 830 & $\delta \mathrm{C}-\mathrm{H}$ substituição aromatic \\
\hline 1580 & $\delta \mathrm{NH}_{2}$ & 739 & $\omega \mathrm{NH}_{2}$ \\
\hline 1512 & $v \mathrm{C}-\mathrm{C}$ aromatic & & \\
\hline
\end{tabular}

$v$ (deformação axial ou estiramento); $v_{\mathrm{a}}$ (deformação axial assimétrica); $v_{\mathrm{s}}$ (deformação axial simétrica); $\delta_{\mathrm{s}}$ (deformação angular simétrica); $\delta_{\mathrm{a}}$ (deformação angular assimétrica). 


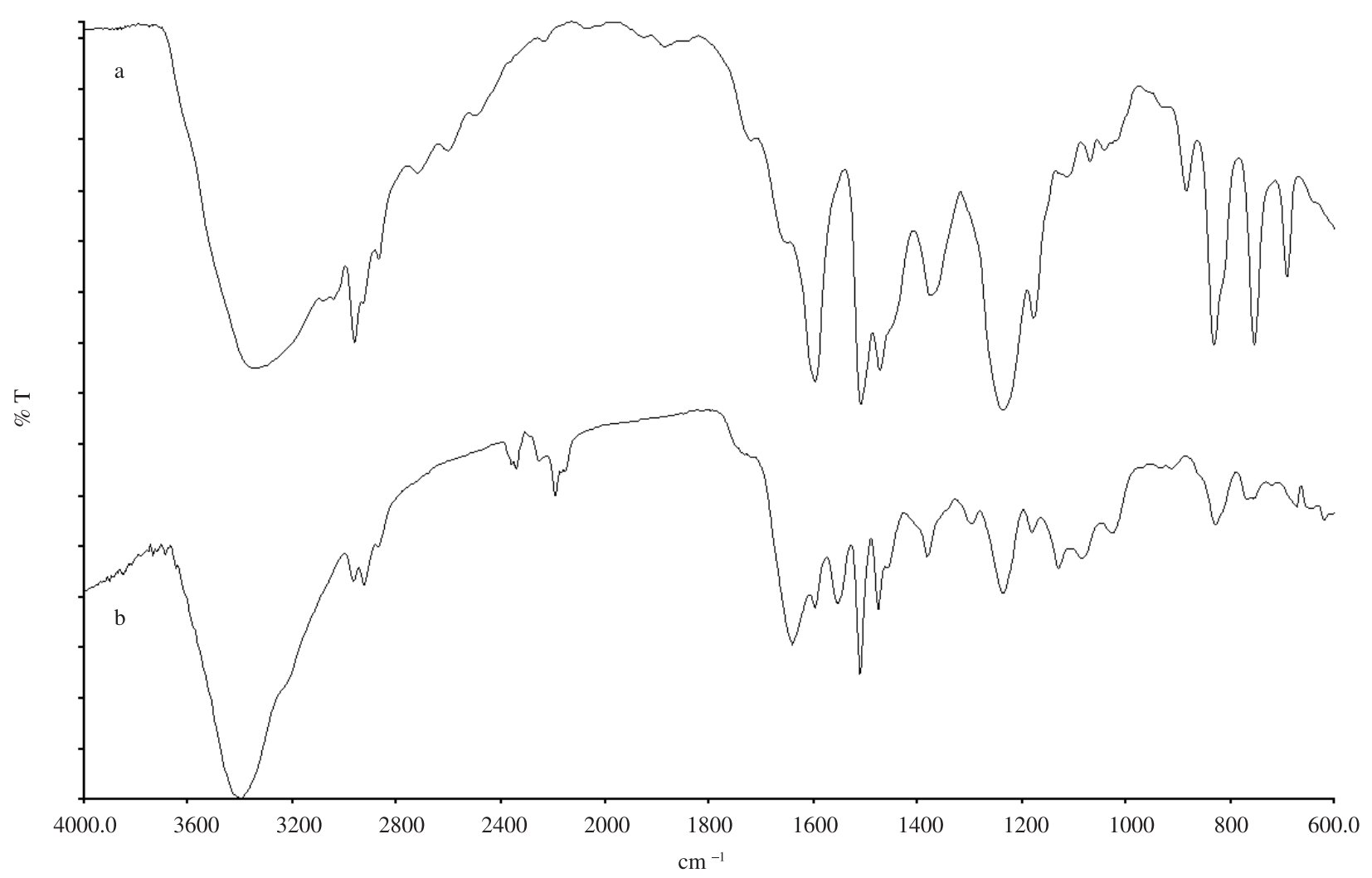

Figura 2. Espectro FT-IR do pirolisado: a) obtido em bico de Bunsen: Bisfenol A e b) pirólise controlada a $340{ }^{\circ} \mathrm{C} / 10$ min: Resina epoxídica, grupos ciano e amínicos e/ou amídicos.

Tabela 2. Bandas características da pirólise do "prepreg" V01X, atribuídas aos modos vibracionais típicos (Figura 2a).

\begin{tabular}{cc}
\hline $\mathbf{N}^{\mathbf{0}}$ de onda $\left(\mathbf{c m}^{-1}\right)$ & Atribuição \\
\hline 3349 & $\vee$ OH \\
1595,1513 e 1474 & $\nu$ C-C aromática \\
1237 e 1178 & $\nu$ C-O \\
761 & $\delta$ C-H substituição aromática \\
\hline
\end{tabular}

$v$ (deformação axial ou estiramento); $\delta$ (deformação angular).

Tabela 3. Bandas características da pirólise a $340{ }^{\circ} \mathrm{C} / 10 \mathrm{~min}$ do "prepreg" V01X, atribuídas aos modos vibracionais típicos (Figura 2b).

\begin{tabular}{|c|c|c|c|}
\hline $\begin{array}{c}\mathbf{N}^{\mathbf{0}} \text { de Onda } \\
\left(\mathrm{cm}^{-1}\right)\end{array}$ & Atribuição & $\begin{array}{c}\mathrm{N}^{\mathbf{o}} \text { de onda } \\
\left(\mathrm{cm}^{-1}\right)\end{array}$ & Atribuição \\
\hline 3401 & $\begin{array}{c}\vee \mathrm{OH} \mathrm{e} / \mathrm{ou} \\
\vee \mathrm{NH}\end{array}$ & 1511 & $\begin{array}{c}\text { v C-C } \\
\text { aromático }\end{array}$ \\
\hline 2968 & $\mathrm{v}_{\mathrm{a}} \mathrm{CH}_{3}$ & 1383 & $\mathrm{Ss} \mathrm{CH}_{3}$ \\
\hline 2928 & $v_{\mathrm{a}} \mathrm{CH}_{2}$ & 1237 & $v \mathrm{C}-\mathrm{O}$ \\
\hline 2194 & $\vee \mathrm{C} \equiv \mathrm{N}$ & 830 & $\begin{array}{c}\delta \mathrm{C}-\mathrm{H} \\
\text { substituição } \\
\text { aromática }\end{array}$ \\
\hline 1640 & $\begin{array}{c}\nu \mathrm{C}=\mathrm{O} \text { e e } \delta \\
\mathrm{NH}\end{array}$ & 739 & $\omega \mathrm{NH}$ \\
\hline
\end{tabular}

$v$ (deformação axial ou estiramento); $v_{\mathrm{a}}$ (deformação axial

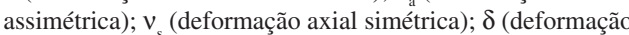
angular); $\omega$ (deformação angular fora do plano).

Para confirmar a presença de cianoguanidina, que é solúvel em água, fez-se um tratamento com água a quente e o resíduo obtido foi analisado e apresentado na Figura $3 b$ que, ao ser comparado com dados da literatura, confirma a presença de cianoguanidina ${ }^{[30]}$.

A análise por FT-IR do material, após a cura no DMTA (Figura $1 \mathrm{~b}$ ) por meio da técnica de pastilha de $\mathrm{KBr}$, permite observar as seguintes alterações espectrométricas quando comparada à análise do mesmo material sem cura (Figura 1a): desaparecimento da banda $2204 \mathrm{~cm}^{-1}$, sugerindo consumo do grupo ciano do agente de cura cianoguanidina; aparecimento da absorção em $1747 \mathrm{~cm}^{-1}$ $(v \mathrm{C}=\mathrm{O})$, sugerindo formação de grupo éster devido à reação de cura; diminuição da intensidade da banda em $1640 \mathrm{~cm}^{-1}$ do agente de cura, sugerindo consumo do grupo $\mathrm{NH}_{2}$ devido ao surgimento de uma banda em $1602 \mathrm{~cm}^{-1}$ que pode indicar mudança de vizinhança de estrutura aromática da resina epoxídica; deslocamento da banda em $1245 \mathrm{~cm}^{-1}$ ( $v$ C-O éter) para $1238 \mathrm{~cm}^{-1}$ e alargamento, indicando mudança da vizinhança dos grupos C-O da resina epoxídica e/ou formação de grupos C-O de éster; desaparecimento da banda em $917 \mathrm{~cm}^{-1}$ indicando consumo do grupo epóxido terminal da resina epoxídica.

Conclui-se que este conjunto de evidências confirma a ocorrência de reação de cura com a formação de grupos éster.

A análise do material após a cura (Figura 4), por meio da técnica fotoacústica FT-IR (PAS), permite observar as seguintes alterações espectrométricas quando comparada à análise do mesmo material sem cura: variação de formato e aumento da intensidade da banda em torno de $3350 \mathrm{~cm}^{-1}$, indicando a formação de grupos $\mathrm{OH}$ ou $\mathrm{NH}$; desaparecimento da banda em $2164 \mathrm{~cm}^{-1}$ e consumo do grupo ciano do agente de cura cianoguanidina; aparecimento da banda em $1747 \mathrm{~cm}^{-1}(\mathrm{v} \mathrm{C}=\mathrm{O})$ indicando a formação de grupo éster, devido à reação de cura; diminuição da banda em $1608 \mathrm{~cm}^{-1}$ que pode sugerir mudança de vizinhança de estrutura aromática da resina epoxídica; diminuição da banda em $1245 \mathrm{~cm}^{-1}$ ( $\vee \mathrm{C}-\mathrm{O}$ éter) indicando mudança da vizinhança dos grupos $\mathrm{C}-\mathrm{O}$ da resina epoxídica; desaparecimento da banda em $917 \mathrm{~cm}^{-1}$ indicando consumo do grupo epóxi terminal da resina epoxídica. 


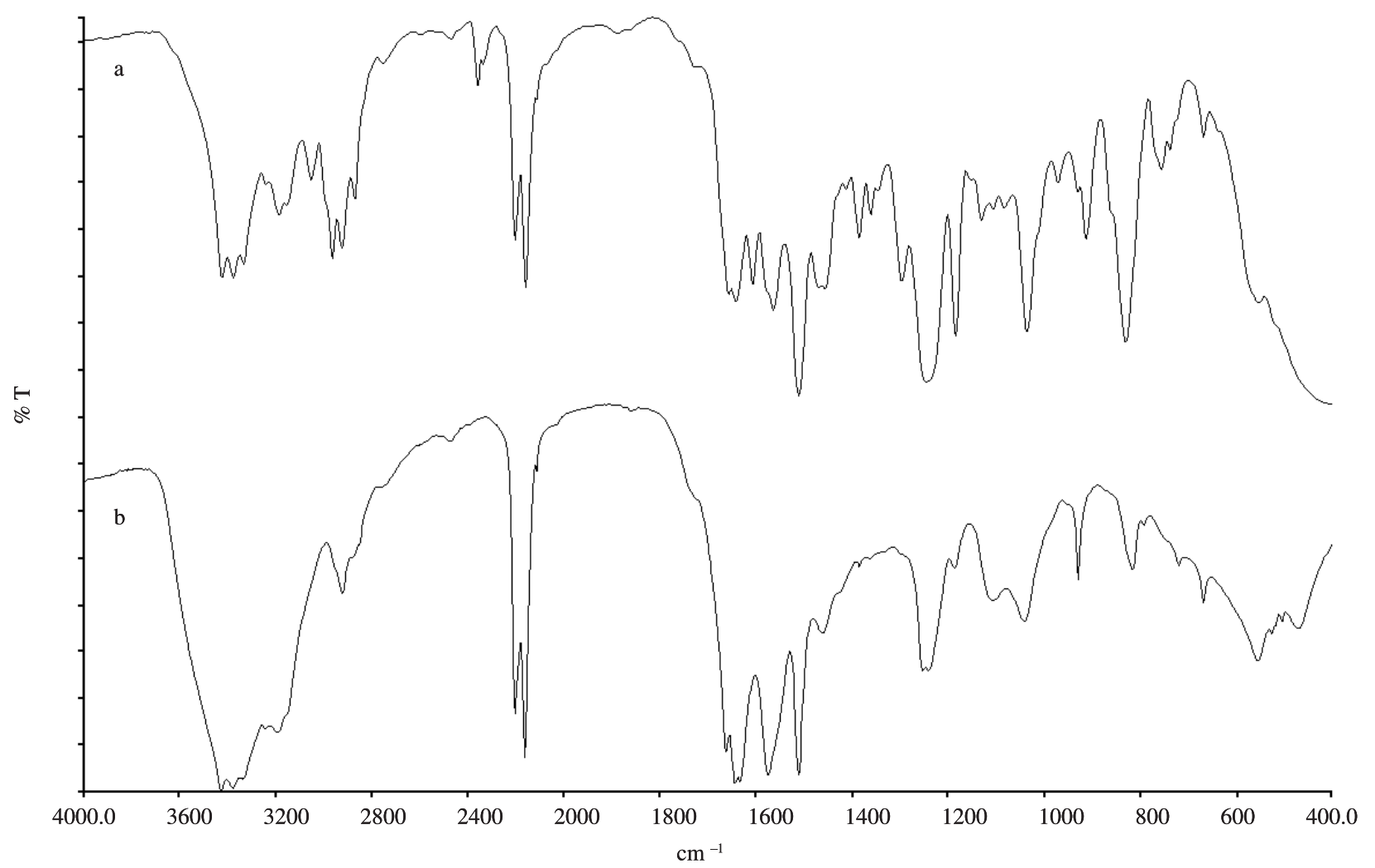

Figura 3. Espectro FT-IR do resíduo obtido após tratamento: a) com metanol: Resina epoxídica e cianoguanidina e b) com água: Cianoguanidina.

Tabela 4. Bandas características do resíduo de "prepreg" V01X após ser tratado com solvente, atribuídas aos modos vibracionais típicos (Figura 3a).

\begin{tabular}{|c|c|c|c|}
\hline $\begin{array}{c}\mathbf{N}^{\mathbf{o}} \text { de onda } \\
\left(\mathrm{cm}^{-1}\right)\end{array}$ & Atribuição & $\begin{array}{c}\mathbf{N}^{0} \text { de onda } \\
\left(\mathrm{cm}^{-1}\right)\end{array}$ & Atribuição \\
\hline $\begin{array}{l}3427,3380 \\
3337,3189\end{array}$ & $v \mathrm{NH}$ & 1563 & $\delta \mathrm{NH}_{2}$ \\
\hline 2967 & $v_{\mathrm{a}} \mathrm{CH} 3$ & 1512 & $\begin{array}{c}\vee \text { C-C aromático } \\
\vee \text { C-C grupo }\end{array}$ \\
\hline 2927 & $v_{\mathrm{s}} \mathrm{CH} 3$ & 1455 & $\begin{array}{l}\text { epoxídico } \\
\text { terminal }\end{array}$ \\
\hline 2205 & $v \mathrm{C} \equiv \mathrm{N}$ & 830 & $\begin{array}{c}\delta \mathrm{C}-\mathrm{H}- \\
\text { substituição } \\
\text { aromática }\end{array}$ \\
\hline 1607 & $\begin{array}{c}v \mathrm{C}-\mathrm{C} \\
\text { aromático }\end{array}$ & & \\
\hline
\end{tabular}

$v$ (deformação axial ou estiramento); $v_{\text {(deformação axial assimétrica); }}$ $\nu_{\mathrm{s}}$ (deformação axial simétrica); $\delta_{\mathrm{s}}$ (deformação angular simétrica); $\delta_{\mathrm{a}}$ (deformação angular).

Este conjunto de dados sugeriu que houve reação de cura completa nesta temperatura, com a formação de grupos éster. Para confirmação, foi incluída a comparação a cura a $130{ }^{\circ} \mathrm{C}$ no DMTA, mostrando que não há mais alterações espectrométricas (Figura 4).

Os resultados da análise FT-IR permitem concluir que a resina que compõe o "prepreg" é epoxídica à base de bisfenol A, e que o provável agente de cura do sistema epoxídico é a cianoguanidina. Comparando-se os dados obtidos por meio das técnicas de transmissão e fotoacústica, as alterações espectrométricas observadas para as amostras analisadas pela segunda técnica, evidenciam melhor as alterações ocorridas nas partes da estrutura referentes ao anel epóxido e grupos aromáticos da resina, enquanto que a técnica por transmissão revela melhor as bandas relativas ao agente de cura.
A análise termogravimétrica teve como objetivo definir a estabilidade térmica e a quantificação do teor de resina no "prepreg", que foi utilizado para corrigir os valores de entalpias de cura obtidos na análise DSC.

Sob atmosfera de nitrogênio (Figura 5) a perda de massa até $300{ }^{\circ} \mathrm{C}$ foi inferior a $1 \%$ sendo mais intensa entre 300 e $500{ }^{\circ} \mathrm{C}$ continuando de forma mais lenta durante a isoterma até estabilizar em 35,2 $\pm 0,5 \%$. A decomposição de resina epoxídica sob atmosfera de nitrogênio pode originar um resíduo carbonáceo, de forma que a perda de massa medida por TG será inferior ao teor de resina presente no "prepreg"[27]. Este resíduo foi quantificado pela análise TG, sob ar e nitrogênio, do "prepreg" V02X que contém a mesma resina epoxídica com fibra de vidro. $\mathrm{O}$ teor de resina sob ar foi de $34 \pm 1 \%$ e sob nitrogênio foi $32 \pm 1 \%$, ou seja, há formação de $2 \%$ de resíduo carbonáceo para a resina epoxídica utilizada nestes "prepregs".

Considerando $100 \%$ de resina, o resíduo seria igual a $6 \%$, consequentemente, o teor de resina no "prepreg" V01X é de $37 \pm 1 \%$.

A partir das curvas DSC não-isotérmicas observa-se 0 deslocamento do pico em direção às temperaturas mais elevadas com aumento da razão de aquecimento.

A entalpia total envolvida no processo de cura da resina (Tabela 5) foi corrigida pelo teor de resina obtido na análise TG e indica que, conforme a razão de aquecimento aumenta, a entalpia do processo de cura diminui. Este fato está relacionado com o encerramento precoce do processo de cura devido ao rápido crescimento da rede tridimensional, que atinge a temperatura de vitrificação e impede a continuação da reação. Isto indica que maiores razões de aquecimento não levam a maiores taxas de cura do material.

As análises DSC isotérmicas (Figura 6) foram realizadas colocando-se as amostras no forno, após ser atingida a temperatura de análise requerida. Devido à metodologia usada, pode-se observar 


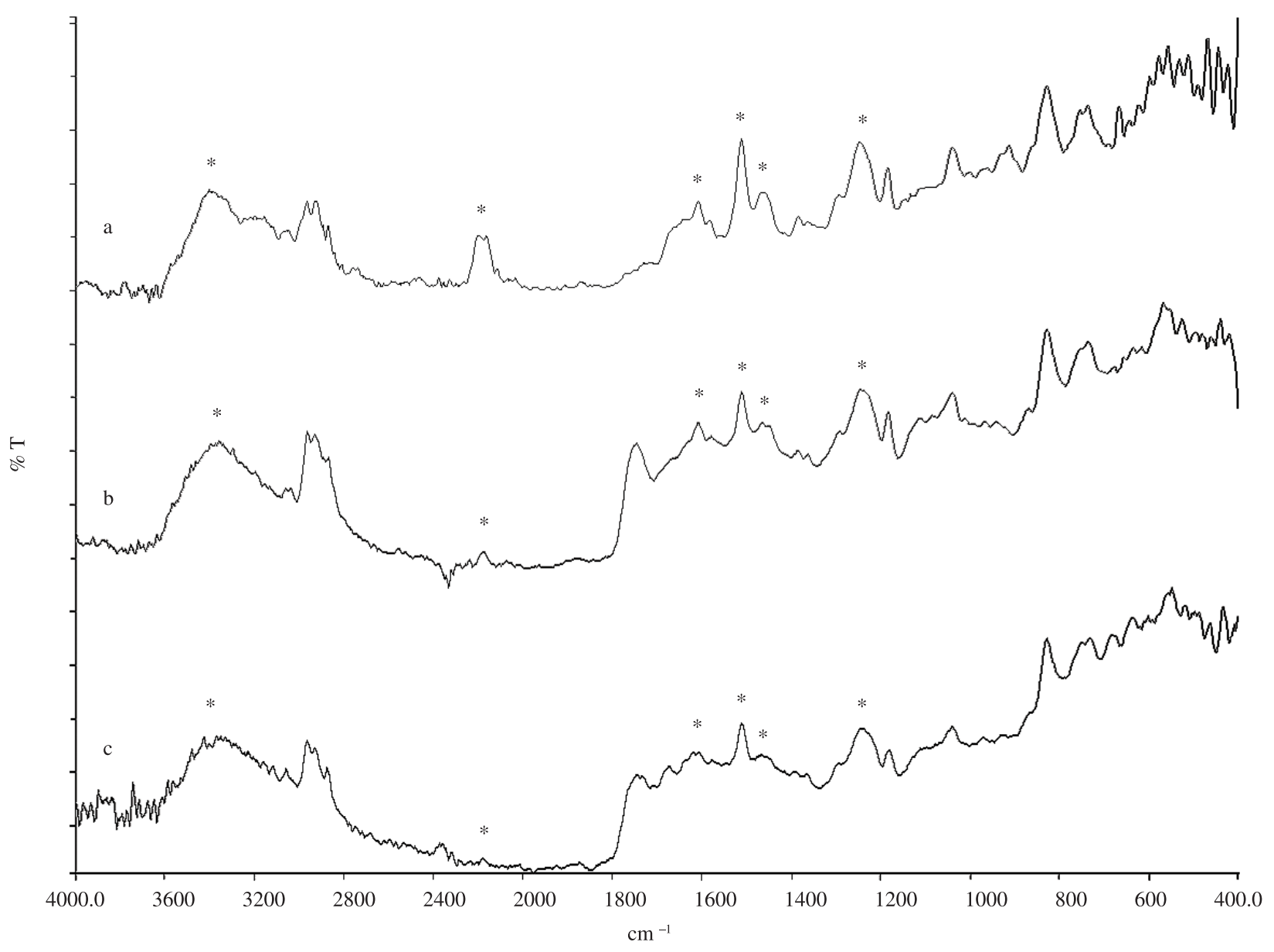

Figura 4. Espectros FT-IR/PAS do "prepreg" V01X: a) (sem cura) e b) (curado a $120^{\circ} \mathrm{C}$ no DMTA) e c) (curado a $130{ }^{\circ} \mathrm{C}$ no DMTA).

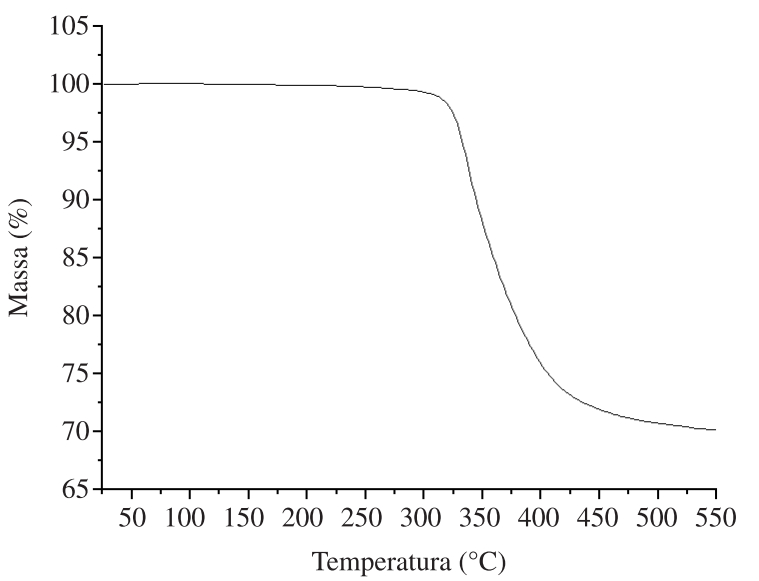

Figura 5. Análise termogravimétrica (TG) do "prepreg" V01X sob atmosfera de $\mathrm{N}_{2}$.

um salto na linha base das corridas, causado pela abertura do forno para a colocação da amostra.

Comparando-se as curvas geradas nas diferentes temperaturas de isoterma, pode-se notar a diferença no comportamento de cura. Confirma-se que o aparecimento do pico depende da temperatura de cura (Tc), onde a intensidade e a largura dos picos se alteram, indicando a necessidade de tempos maiores para completar a cura quando a temperatura diminui. Estes valores (Tabela 5) mostram que o aumento da temperatura de cura (até $130{ }^{\circ} \mathrm{C}$ ) provoca um aumento na entalpia de cura, que se mantém nas demais Tc.

Este fato indica que maiores temperaturas de cura não levam a maiores graus de conversão do material citado. Para compreender melhor estas informações foram realizadas as análises DMTA para verificar como as transições: gelificação e vitrificação, influenciavam no processo de cura.

Observa-se baixa repetitividade nos valores de entalpia na cura isotérmica. Nas temperaturas mais baixas a reação é muito lenta e o final do pico é de difícil identificação. Por outro lado, nas temperaturas mais altas, a reação é rápida, podendo iniciar antes que todo o sistema atinja a temperatura do forno, dificultando a identificação do início do pico.

A simples comparação entre os valores de entalpia de cura obtidos pelos métodos não-isotérmicos e isotérmicos (Tabela 5) indica maiores valores de entalpia para as corridas isotérmicas, demonstrando que, apesar de ocorrer a cura do "prepreg" nas duas condições, o processo de cura não é similar e que, para maiores taxas de conversão, o método isotérmico é o mais indicado.

As amostras do "prepreg" foram submetidas a um segundo aquecimento para se obter os valores das Tg (Tabela 5).

Os baixos valores de $\mathrm{Tg}$ obtidos a $100{ }^{\circ} \mathrm{C}$ indicam que a cura não foi completa quando comparados com as demais temperaturas de cura. Os valores de $\mathrm{Tg}$, tanto para as amostras curadas pelo método não-isotérmico quanto pelo método isotérmico, indicam uma leve tendência de aumento com a temperatura de cura, até um limite onde passam a diminuir, indicando a necessidade de se 


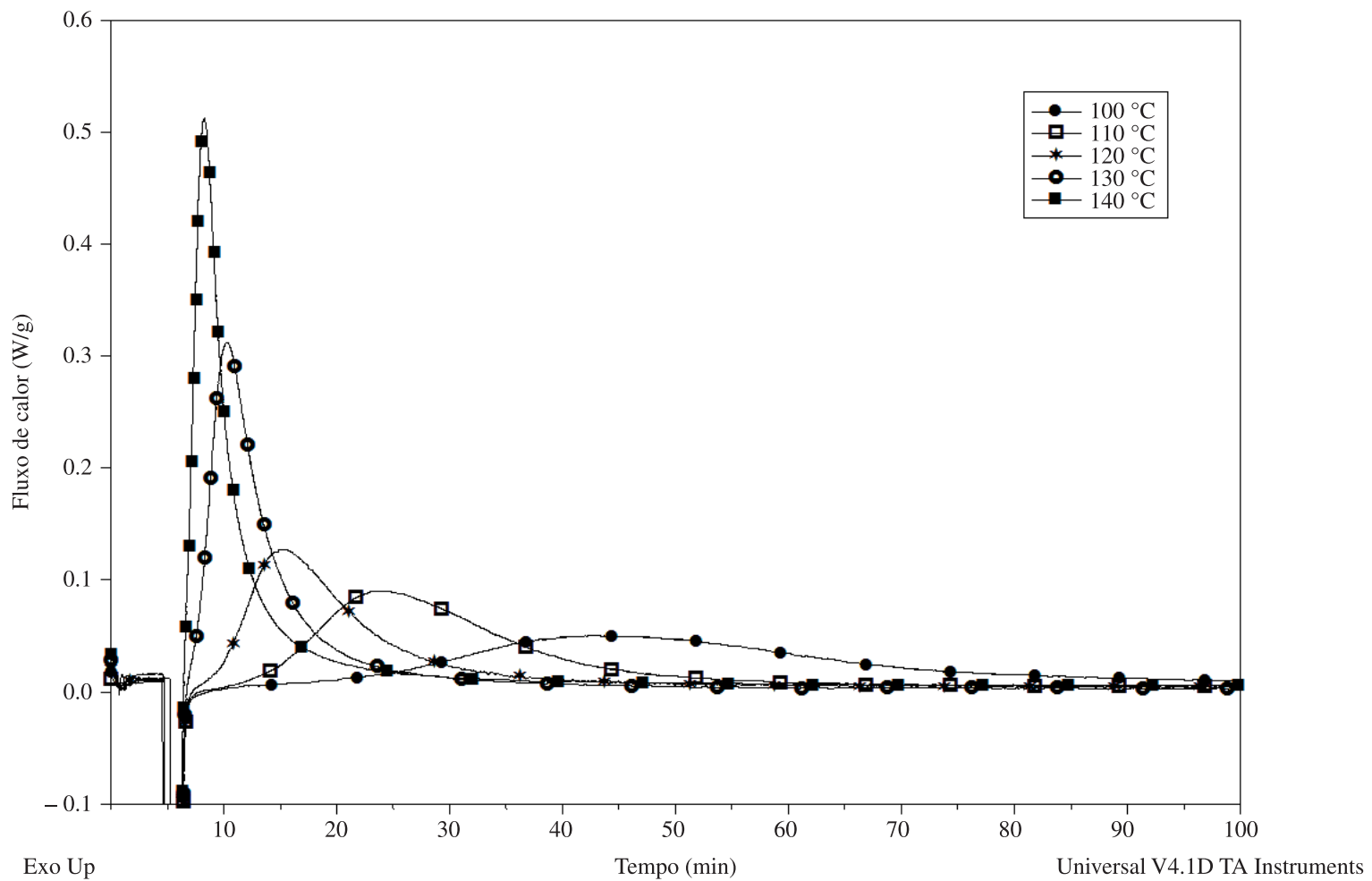

Figura 6. Análise calorimétrica exploratória diferencial (DSC). Curvas da cura isotérmica do "prepreg" V01X.

Tabela 5. Entalpia de cura do "prepreg" e suas respectivas Tg.

\begin{tabular}{cccccc}
\hline \multicolumn{2}{c}{ Método não-isotérmico } & \multicolumn{3}{c}{ Método isotérmico } \\
\hline $\begin{array}{c}\text { Razão de } \\
\begin{array}{c}\text { Aquecimento } \\
\left({ }^{\circ} \mathbf{C} / \mathbf{m i n}\right)\end{array}\end{array}$ & $\begin{array}{c}\Delta \mathbf{H} \\
\left(\mathbf{J . g}^{-1}\right)\end{array}$ & $\begin{array}{c}\text { Tg } \\
\left({ }^{\circ} \mathbf{C}\right)\end{array}$ & $\begin{array}{c}\text { Tcura } \\
\left({ }^{\circ} \mathbf{C}\right)\end{array}$ & $\begin{array}{c}\Delta \mathbf{H} \\
\left(\mathbf{J . g}^{-1}\right)\end{array}$ & $\begin{array}{c}\mathbf{T g} \\
\left({ }^{\circ} \mathbf{C}\right)\end{array}$ \\
\hline 5 & $267 \pm 7$ & $128 \pm 4$ & 100 & $254 \pm 16$ & $113 \pm 5$ \\
10 & $242 \pm 1$ & $128 \pm 2$ & 110 & $277 \pm 8$ & $130 \pm 1$ \\
15 & $223 \pm 3$ & $121 \pm 3$ & 120 & $272 \pm 40$ & $132 \pm 5$ \\
20 & $217 \pm 1$ & $122 \pm 1$ & 130 & $295 \pm 14$ & $134 \pm 2$ \\
& & & 140 & $297 \pm 31$ & $126 \pm 4$ \\
\hline
\end{tabular}

trabalhar dentro de uma faixa de temperatura para realizar a cura de forma a se atingir os maiores valores de $\mathrm{Tg}$.

No início da corrida não-isotérmica dos "prepregs", a viscosidade da resina diminui devido ao aquecimento do corpo-de-prova, que provoca um aumento da mobilidade das cadeias poliméricas dos reagentes, o que pode ser observado por meio do decréscimo do módulo de armazenamento (E') (Figura 7a) e do módulo de perda (E”) (Figura 7b). Posteriormente, inicia-se a polimerização com o entrecruzamento de cadeias e, consequentemente, ocorre o aumento de viscosidade da resina, refletido pelo súbito aumento de E' e E" (Figuras 7a, b).

Na corrida não-isotérmica pode-se observar o aumento de E' (Figura 7a), que indica o início do processo de cura do material à temperatura aproximada de $142{ }^{\circ} \mathrm{C}$ e se estende até a temperatura de $225{ }^{\circ} \mathrm{C}$. Simultaneamente, durante o processo de cura, na curva E" (Figura 7b) ocorre o aparecimento de dois picos nítidos e diferentes, os quais apresentam os máximos às temperaturas de aproximadamente $148{ }^{\circ} \mathrm{C}$ e $163{ }^{\circ} \mathrm{C}$; estes picos também estão presentes na curva tan $\delta$ (Figura $7 \mathrm{c}$ ) em $147{ }^{\circ} \mathrm{C}$ e $161^{\circ} \mathrm{C}$. Estes picos são atribuídos aos processos de mudança de estrutura denominados gelificação e vitrificação.
Nas corridas isotérmicas verifica-se que, em todas as isotermas, ocorre a cura evidenciado pelo aumento de E' (Figura 8a) . Nas curvas E" e tan $\delta$ (Figuras 8b, c), o pico de vitrificação ocorre apenas nas temperaturas de isoterma a 100,110 e $120^{\circ} \mathrm{C}$. A diminuição do valor final de E' (Figura 8a) e o aumento no $\tan \delta$ (Figura 8c) confirmam a ausência da vitrificação nas Tc mais altas.

O pico de gelificação, tanto no E" como no tan $\delta$ (Figuras 8b, c), aparentemente, não é afetado pela mudança de Tc, enquanto o pico de vitrificação (Figuras 8b, c) diminui de intensidade conforme aumenta a Tc, não podendo mais ser identificado a partir de $130^{\circ} \mathrm{C}$.

Observou-se que a ocorrência da gelificação não está relacionada à temperatura de cura enquanto a vitrificação é fortemente dependente desta.

As amostras dos "prepregs" foram submetidas a um segundo aquecimento a $3{ }^{\circ} \mathrm{C} / \mathrm{min}$, para se obter os valores de Tg a partir dos valores de $\tan \delta$ no máximo do pico (Tabela 6 ). Verifica-se que nas Tc até $120{ }^{\circ} \mathrm{C}$ as $\mathrm{Tg}$ aumentam e, a partir de $130{ }^{\circ} \mathrm{C}$ diminuem. $\mathrm{O}$ "prepreg" curado a $120^{\circ} \mathrm{C}$ é o que apresenta maior valor de $\mathrm{Tg}$. A informação das temperaturas em que ocorre o processo de gelificação seguido de vitrificação permite recomendar a faixa de temperatura ideal de 120 a $130{ }^{\circ} \mathrm{C}$ para efetuar a manufatura de peças, o que permitiria que a cura ocorresse com a menor interferência possível do processo de vitrificação, porém com o maior grau de conversão do material.

Tabela 6. Temperaturas de $\mathrm{Tg}$ das amostras curadas em isotermas $(\tan \delta)$.

\begin{tabular}{cc}
\hline T de cura $\left({ }^{\circ} \mathbf{C}\right)$ & Tg médio $\left({ }^{\circ} \mathbf{C}\right)$ \\
\hline 100 & $142,4 \pm 0,2$ \\
110 & $150,0 \pm 0,2$ \\
120 & $153,8 \pm 1,2$ \\
130 & $150,7 \pm 1,0$ \\
140 & $148,3 \pm 2,0$ \\
150 & $148,8 \pm 2,0$ \\
\hline
\end{tabular}




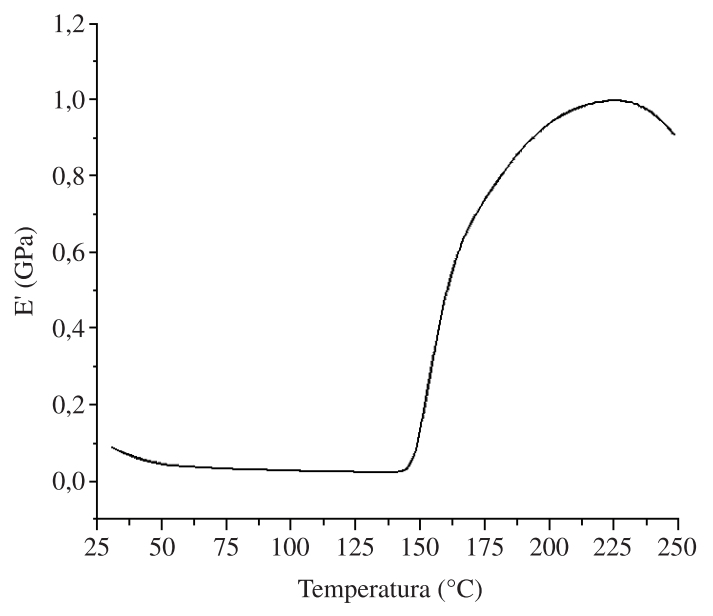

(a)

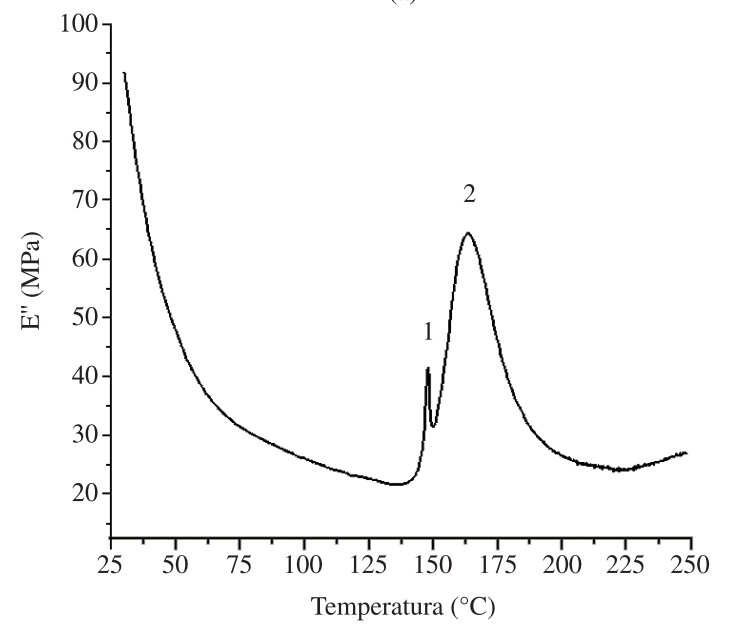

(b)

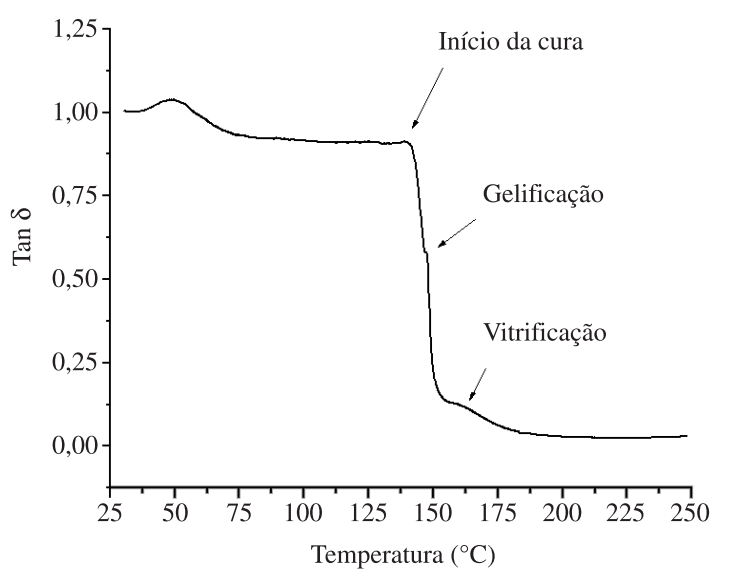

(c)

Figura 7. Curvas DMTA não-isotérmicas do "prepreg" V01X: a) E' $\times$ Temperatura b) E" $\times$ Temperatura e c) $\tan \delta \times$ Temperatura.

\section{Conclusão}

A análise por FT-IR de transmissão do "prepreg", antes da cura, permitiu a identificação do agente de cura, cianoguanadina. Comparando-se os dados obtidos por meio das técnicas de transmissão e fotoacústica, as alterações espectrométricas observadas para as amostras analisadas pela segunda técnica evidenciam melhor

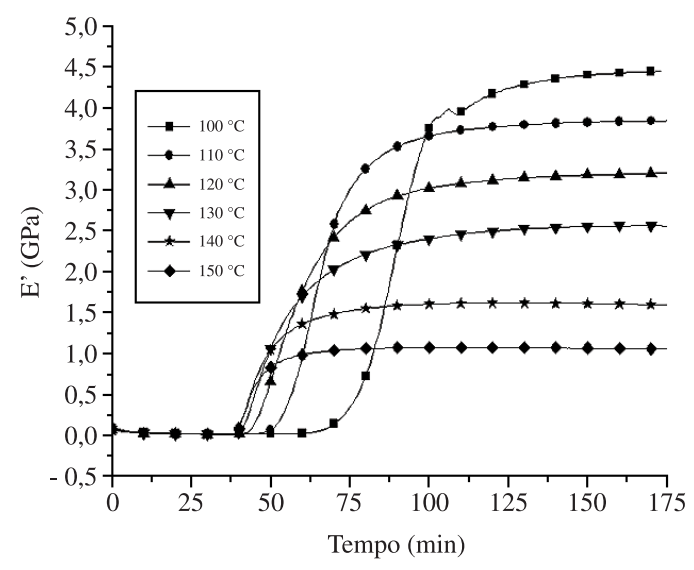

(a)

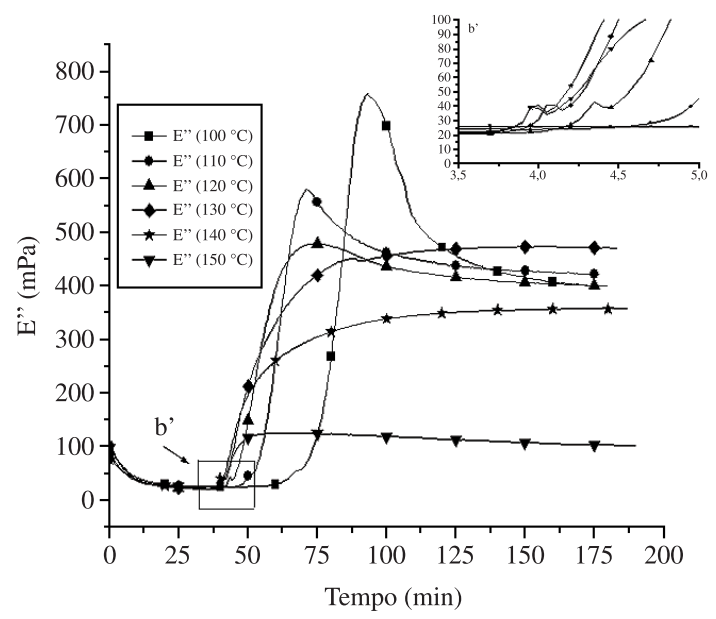

(b)

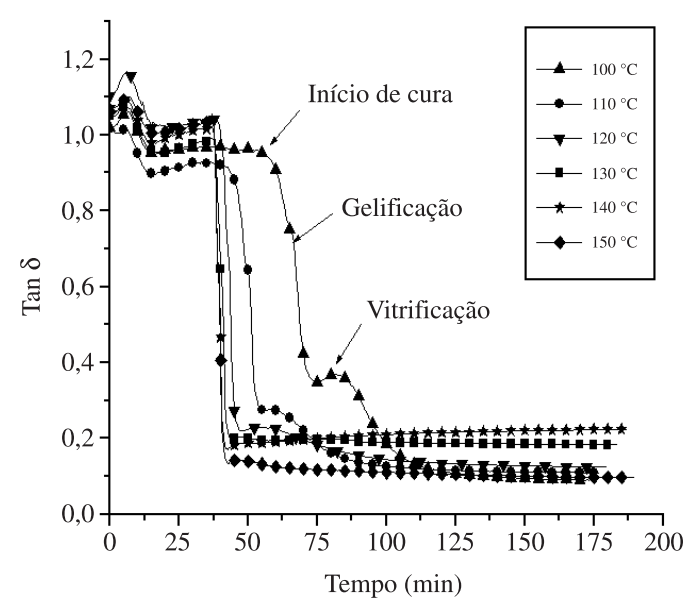

(c)

Figura 8. Curvas DMTA isotérmicas do "prepreg" V01X: a) E' $\times$ Tempo; b) E" $\times$ Tempo e c) $\tan \delta \times$ Tempo.

as alterações ocorridas nas partes da estrutura referentes ao anel epóxido e grupos aromáticos da resina.

$\mathrm{O}$ estudo de análise térmica permitiu concluir que maiores temperaturas de cura e maiores taxas de conversão não implicam em melhores condições de reação. Neste trabalho, demonstrou-se que a temperatura de cura ideal do "prepreg" V01X encontra-se entre 120 a $130{ }^{\circ} \mathrm{C}$ e que a ocorrência do fenômeno de vitrificação durante 
o processo de cura influencia no grau de conversão do material. Também foi possível evidenciar que existe um limite de razão de aquecimento, acima do qual a Tg também é prejudicada. Logo, é possível melhorar a qualidade dos produtos obtidos mediante um maior controle do processo de cura.

\section{Referências Bibliográficas}

1. ASM International Comitee - "Engineered Materials Handbook". in: ASM International, v.1: Composites (1987).

2. Calado, V. M. A. \& Advani, S. G. - Thermoset Resin Cure Kinetics and Rheology. In: Processing of Composites, R.S. Davé \& A.C. Loos, Washington, USA (2000)

3. Jiawu, G.; Kui, S. \& Mong, G. Z. - Termochim. Acta, 352, p.153 (2000). http://dx.doi.org/10.1016/S0040-6031(99)00460-8

4. Romão, B. M. V.; Diniz, M. F.; Azevedo, F. M. P.; Lourenço, V. L.; Pardini, L. C.; Dutra; R. C. L. \& Burel, F. - Polímeros, 13, p.173 (2003).

5. Sugita, T. \& Ito, M. - Bull. Chem. Soc. Japan, 38, p.1620 (1965). http:// dx.doi.org/10.1246/bcsj.38.1620

6. Sales, R. C. M.; Diniz, M. F.; Dutra, R. C. L.; Thim, G. P. \& DibbernBrunelli, D. - J. Appl. Polym. Sci., 117, n.2, p.664 (2010). http://dx.doi. org/10.1002/app.31953

7. Romão, B. M. V.; Dutra, R. C. L.; Diniz, M. F. \& Ribeiro, A. P.- Pesq. Naval, 14, p.273 (2001).

8. Dutra, R. C. L. - "Modificação de fibras de polipropileno com EVA funcionalizado", Tese de Doutorado, Universidade Federal do Rio de Janeiro, Brasil (1997).

9. Laza, J. M.; Julian, C. A.; Larrauri; E.; Rodriguez, M. \& Leon, L. M. - Polymer, 40, p.35 (1998). http://dx.doi.org/10.1016/S00323861(98)00217-1

10. Prime, R. B. - "Thermosets", in: Thermal Characterization of Polymeric Materials, cap.5, Turi, E. A. (ed.), Academic Press, New York (1981).

11. Gillham, J. K. - "Torsional Braid Analysis (TBA) of Polymers", in: Developments in Polymer Characterization, v.3, cap.5, Dawkins, J. V. (ed.), Applied Science Publishers Ltd., New Jersey (1982).

12. Sickfeld, J. \& Mielke, W. - Prog. Org. Coat., 12, p.27 (1984). http:// dx.doi.org/10.1016/0033-0655(84)80003-5

13. David, J. B. \& John, C. W. - "Curing Epoxy Resins", in: Engineered Materials Handbook, Composites, v.1, p.654, ASM International, USA (1987).
14. Kontou, E.; Spathis, G. \& Theocaris, P. S. - J. Polym. Sci. Polym. Chem. Ed., 23, p.1493 (1985). http://dx.doi.org/10.1002/pol.1985.170230521

15. Shim, S. B.; Seferis, J. C.; Eom, Y. S. \& Shim, Y. T. - Termochim. Acta, 291, p.73. (1997). http://dx.doi.org/10.1016/S00406031(96)03084-5

16. Cadenato, A.; Salla, J. M.; Ramis, X.; Morancho, J. M.; Marroyo, L.M. \& Martin, J. L. - J. Therm. Anal., 49, p.269 (1997). http://dx.doi.org/10.1007/ BF01987448

17. Wingard, C. D. - Termochim. Acta 357-358', p.293 (2000).

18. Paiva, J. M. F.; Mayer, S.; Cândido, G. M. \& Rezende - Polímeros, 16, n.1, p.79. (2006).

19. Sun, L.; Negulescu, I.; Pang, S. \& Sterling, A. - J. Adhes., 82, p.161. (2006). http://dx.doi.org/10.1080/00218460600559573

20. Xie, M.; Zhang, Z.; Gu, Y.; Li, M. \& Su, Y. - Termochim. Acta, 487, p.8 (2009). http://dx.doi.org/10.1016/j.tca.2009.01.001

21. Hofmann, K. \& Glasser, W. G. - Termochim. Acta, 166, p.169 (1990). http://dx.doi.org/10.1016/0040-6031(90)80179-3

22. Lange, J.; Altmann, N.; Kelly, C. T. \& Halley, P. J. - Polymer, 41, p.5949 (2000). http://dx.doi.org/10.1016/S0032-3861(99)00758-2

23. Kim, J. S. \& Lee, D. G. - J. Compos. Mater., 30, n.3 (1996).

24. Murayama, T. - "Dynamic Mechanical Analysis of Polymeric Material", v.1, Elsevier Scientific Publishing Co., New York (1978)

25. Atkinson, K. E. \& Jones, C. - J. Adhes., 56, p.247 (1996). http://dx.doi. org/10.1080/00218469608010511

26. Lange, J. \& Manson, J. A. - Polymer, 37 , n.26, p.5859 (1996). http:// dx.doi.org/10.1016/S0032-3861(96)00441-7

27. Yee, R. Y. \& Stephens, T. S. - Termochim. Acta, 272, p.191 (1996). http://dx.doi.org/10.1016/0040-6031(95)02606-1

28. Hummel, D. O. et al. - "Atlas of Polymer and Plastics Analysis", v.III, Weinheim, V.C.H. (1981). 2792.

29. Hummel, D. O. et al. - "Atlas of Polymer and Plastics Analysis", v.III, Weinheim, V.C.H. (1981). 5912.

30. Hummel, D. O. et al. - "Atlas of Polymer and Plastics Analysis", v.III, Weinheim, V.C.H. (1981). 6432

Enviado: $11 / 06 / 10$

Reenviado: 18/09/11 Aceito: 10/11/11 